\title{
Effects of Local Configuration on the Flow in the Circle of Willis
}

\author{
J. H. Page ${ }^{1 *}$, G. X. Wu ${ }^{1}$, F. T. Smith ${ }^{2}$ \\ ${ }^{1}$ Department of Mechanical Engineering, UCL, Torrington Place, London, WC1E 7JE, UK \\ ${ }^{2}$ Department of Mathematics, UCL, Gower Street, London, WC1E 6BT, UK \\ Email: j_page@meng.ucl.ac.uk, gx_wu@meng.ucl.ac.uk, frank@math.ucl.ac.uk
}

\begin{abstract}
The circle of Willis is a confluence of large arteries which form a ring-like, three-to-six junction. The right and left internal carotid arteries and the basilar artery carry blood up the neck and meet a circular arrangement of three communicating arteries at the base of the cerebrum. Six cerebral arteries then depart from the circle of Willis towards different areas of the brain. The circle of Willis plays a pivotal role in allowing the correct blood distribution in the cerebrum. The presence of the communicating arteries provides routes along which blood may flow in order to moderate or effect changes in the efferent flux distribution, in response to variations in the supply of and local demands for blood[1]. The anterior communicating artery is a common site for the occurrence of cerebral aneurysms, an abnormal bulge in the arterial wall which grows over time[2]. Such cerebral aneurysms can eventually rupture leading to a subarachnoid haemorrhage[2]. It is widely thought that forces exerted by the blood on the arterial wall, such as wall pressure and wall shear stress, make a significant contribution to the growth of aneurysms[3].

In this paper, we examine the variation in wall shear stress along a surface common to the anterior cerebral and anterior communicating arteries. A two-dimensional representation of the circle of Willis is used. The Navier-Stokes equations are solved using a formulation of the finite volume method with pulsatile inputs and resistance outputs. The results of velocity field and wall shear stress are provided.
\end{abstract}

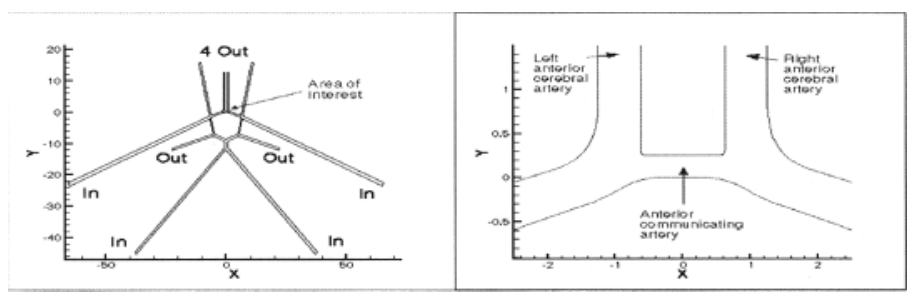

Figure: the circle of Willis model with marked inputs, outputs and area of interest

\section{REFERENCES}

1. Hillen B, Hoogstraten $\mathrm{H} \mathrm{W}$, Post L. A mathematical model of the flow in the circle of Willis. $J$ Biomech, 1986; 19: 187-194

2. Yasargil M G. Microneurosurgery, 2 Thieme, 1984

3. Valencia A A, Guzmán A M, Finol E A, Amon C H. Blood flow dynamics in saccular aneurysm models of the basilar artery. J Biomech Eng, 2006; 128: 516-526 